\title{
Jitter Spectral Extraction for Multi-gigahertz Signal
}

\author{
Chee-Kian Ong, Dongwoo Hong, Kwang-Ting (Tim) Cheng, Li-C Wang \\ Department of Electrical and Computer Engineering \\ University of California, Santa Barbara, CA 93106, USA \\ Email: ckong@ece.ucsb.edu
}

\begin{abstract}
In this paper, we propose a method for extracting the spectral information of a multi-gigahertz jittery signal. This method may utilize existing on-chip single-shot period measurement techniques to measure the multi-gigahertz signal periods for spectral analysis. This method does not require an external sampling clock, nor any additional measurement beyond existing techniques. Experimental results show that this analysis method can accurately estimate the amount and frequencies of periodic and random jitter of a multi-gigahertz signal.
\end{abstract}

\section{INTRODUCTION}

With marketing gimmicks pushing for higher processor speed and industry demanding faster and more reliable electronic communications, one important engineering task is to ensure that all the signals arrive at their destination at the appropriate time.

The demand for greater speed has pushed the processor operating frequency to the Radio Frequency (RF) hitting the multi-gigahertz range and edging upward. Systems performance limited by parallel-bus data transfer has motivated engineers to look for alternative expandable communication architecture, including multi-gigahertz serial communication architectures (e.g. Infiniband, 3GIO, SONET, SATA). Video-in-demand and other information-hungry applications have also propelled networks into the Gigabit Ethernet area. All these systems operate in the multigigahertz range, and to achieve reliable operation for these systems requires substantial understanding of timing jitter characteristics [1].

Jitter is the deviation of a signal event from its ideal position. Jitter affects different systems in different ways, and it can be introduced by every circuit element used to generate, convey or receive signals. Understanding the type and amount of jitter introduced by each element of a system is crucial for predicting overall system performance. For example, some systems can tolerate long-term drift in a signal frequency, whereas the same drift would be catastrophic if it occurred within two successive cycles.

However, most existing jitter analysis techniques have centered upon histogram-based analysis (including data-eye diagram analysis) [2,3,4,5,6]. Histogram-based analysis conceals the jitter spectral information and thus does not reflect well on performance of systems which readily adapt to some degree of drift in the signal frequency within a given period (for example a pair of serial communication transceivers).

The $\Delta \phi$ method proposed in [7] could extract the peak-topeak amplitude and RMS of a sinusoid jitter in a $200 \mathrm{MHz}$ clock signal using external ATE. The $\Delta \phi$ method has been extended [8] to perform jitter spectral analysis on a $10 \mathrm{GHz}$ clock using frequency division. Both methods depend on external equipment and external reference clocks to ensure accuracy. However, the jitter characteristics of a signal may be more complex than a sinusoid. As stated in [9], assuming the jitter characteristic with a double-delta power density function (PDF) is inaccurate in estimating deterministic and random jitters, and hence, the performance of the system. Intuitively, double-delta PDF would be the convolution of a sinusoidal function with a random Gaussian-distribution function (will be shown later in Figure 1).

The method reported in [10] could perform jitter spectral analysis on high-speed signals. The principle of this method is to reconstruct a predetermined test pattern (i.e. a sequence of 0's and 1's) and perform spectral analysis on it. First the test pattern is repeatedly transmitted, and multiple timing measurements are taken from the first edge to the other edges of the test pattern. Thus, each pattern edge has a timing distribution to indicate its occurrence with respect to the first edge. Then, the test pattern is reconstructed based on the timing distribution of each pattern edge, and spectral analysis can be performed on the reconstructed pattern.

This method requires measurement covering a very wide range of timing intervals (ranging from the pattern smallest period to its entire length). This requirement may be too intricate for implementing a single, on-chip timing interval measurement unit. Therefore, multiple timing-measurement units with different resolutions may be required to cover such a wide range of timing measurements. A large amount of memory space is also required for storing the timing distribution of large numbers of test pattern edges. Because of these requirements (multiple timing measurement units and large memory space), this method could only be implemented only by using external equipment. Moreover, this method may not accurately extract the signal jitter spectrum, as the frequency of sinusoidal jitter may not be a multiple of the repeating frequency of the re-constructed test pattern. For such cases, this method would not correctly capture the periodicity of the jitter during the re-construction process of the test pattern.

Today, most schemes would require sampling and measurement mechanisms to perform an accurate spectral analysis. Our proposed jitter spectral analysis technique requires only the measurement mechanism, which could be implemented using existing single-shot period measurement techniques. Instead of generating a repetitive test pattern, the proposed technique only requires the transmission of the signal's smallest period (i.e. a sequence of alternating 1's and 0's "101010..."). The transmission of this sequence allows the technique to extract the random and periodical jitter spectrum information without interference from non-single period transmission (commonly known as the data-dependent jitter). Hence, the timing measurement range requirement is only as large as the peak-to-peak amplitude of total jitter, which is much smaller than the requirement of the technique reported in [10]. In addition, the proposed technique does not require any reference clock to trigger the sampling of the periods. These features make the proposed technique a viable on-chip solution for spectral analysis of signal jitter. 
This paper is organized with the next section giving brief descriptions of types of jitters and explaining how they are represented in a general form. The general single-shot period measurement techniques will also be briefly described in Section 2. Section 3 presents the overview of the analysis technique and explains how the signal period sampling may be implemented using existing single-shot period measurement techniques. Section 4 will present in detail the principle of the spectral analysis technique while section 5 presents the experimental setup and results showing the accuracy of the proposed analysis. We conclude in section 6 , with discussion of the potential uses of this technique.

\section{BACKGROUND}

\section{A. Jitter and its Representation}

In an electronic system, jitter can be generally classified into two categories - (1) non-deterministic/random jitter, and (2) deterministic jitter $[3,4,5,6,9]$. Non-deterministic jitter is best characterized by a random process with Gaussian probability density function (PDF). Many factors contribute to the deterministic jitter (for example, periodic and datadependent jitters). However, we could represent the deterministic jitter with a general process commonly used to describe deterministic analog signal - the sum of sinusoidal signals with different amplitudes and frequencies as follows:

$$
f_{D}=\sum_{i=1}^{n} a_{i} \sin \left(\omega_{i} t\right)
$$

where $n$ is the number of sinusoidal functions found in the deterministic process. $a_{i}$ and $\omega_{i}$ are the $i^{\text {th }}$ sinusoidal jitter's peak and frequency, respectively.

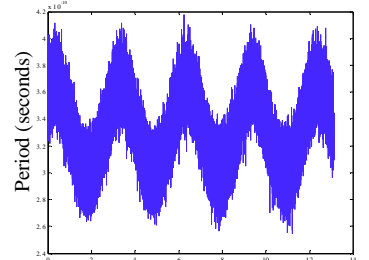

Time (seconds)

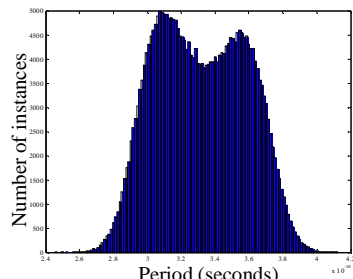

Period (seconds)

Figure 1 Signal periods with a sinusoidal and a random jitter.

It is important to represent jitter in a general form, since it has been shown in [9] that the previous jitter distribution assumption does not reflect well in actual testing. Figure 1 shows the period transient plot (left) of a signal whose jitter characteristic is the sum of a sinusoidal process and a random Gaussian distribution process. The signal period distribution is shown on the right diagram of Figure 1. Note that the distribution diagram reflects that of a double delta distribution function mentioned in [9].

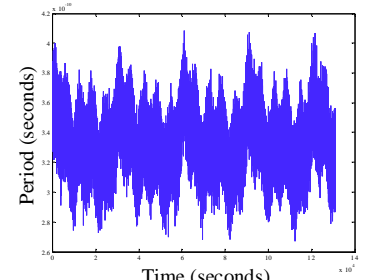

Figure 2 Signal Periods with three sinusoidal jitters and a random jitter.

Figure 2 shows the periods and its distribution of a signal whose jitter function is the sum of a random Gaussian distribution process and three sinusoid processes. Note that the distribution diagram on the right could not visibly reflect the three sinusoidal jitter components in the signal. It appears more like a Gaussian distribution whose standard deviation, $\sigma$, is larger than the random Gaussian process in the signal. The histogram analysis may not be the best choice for determining the performance of a system whose characteristic could endure the slow frequency drift caused by the three sinusoidal jitters. Hence, it is important to extract the signal jitter in the general form before making any conclusive system performance analysis [1].

\section{B. Single-shot Period Measurement}

This section presents the overview of the existing singleshot period measurement techniques that may be implemented in the spectral analysis technique as the Time Measuring Unit (TMU). Generally, these techniques can be classified into two categories: (1) the Vernier Delay Line (VDL) and (2) the dual slope techniques [11, 12].

The VDL category includes the Time-to-Digital converters [13, 14], the Component Invariant VDL [15, 16], and the multiple oscillators with different frequencies $[17,18$, 19, 20]. All these VDL techniques revolve around the Vernier principle that has been used in the Vernier caliber for measuring distance [21]. The Vernier principle is based on the principle that two events begin at two different points as shown in Figure 3. The first event would begin its count at a fixed interval $P_{l}$. The second event would begin its count at a smaller fixed interval $P_{2}$. Both counts would reach a coincident point, which stops the counts. Thus, each event generates a product of its respective count Count $_{n}$ and interval $P_{n}$. The distance between the two events can be determined by the difference of the two products as depicted in Figure 3.

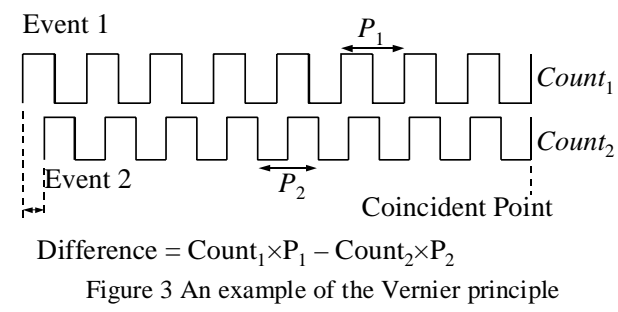

Among these VDL techniques, some have claimed accuracy measured in sub-picoseconds. Thus, we may utilize the existing single-shot period measurement techniques to realize our Time Measuring Unit (TMU) to perform period measurement on multi-gigahertz signal for our proposed technique on jitter spectral analysis.

\section{Overview of Proposed Technique}

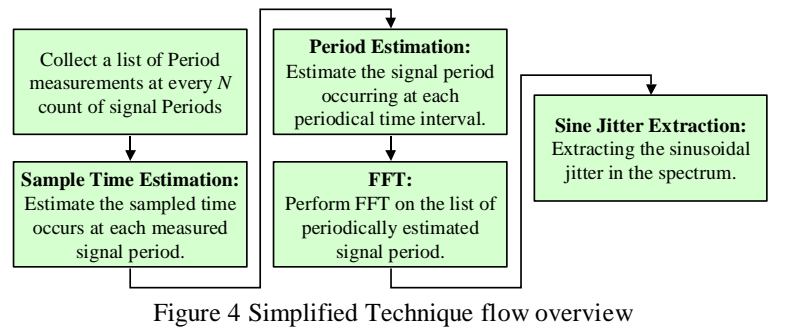

To extract any signal spectral information, the signal amplitude needs to be periodically sampled over a given time. It is no exception for spectral analysis on the signal's jitter. However, one great challenge is to sample periods of a 
multi-gigahertz signal at absolute periodic intervals. A jitterfree sampling clock signal would be required to trigger the TMU to perform period measurement.

\section{A. Principle of the Technique}

An overview of the proposed technique is illustrated in Figure 4. The technique does not require a sampling clock signal to perform spectral analysis on the signal. Instead, it collects a list of signal periods by measuring each signal period width on every $N$ count of signal periods as elaborated in Figure 5. With each sampled period value, the technique has a simple Sample Time Estimation procedure to estimate the time $T_{n}$ at which each period is sampled. With this procedure, every sampled period can be associated with an estimated sampling time $T_{n}$ as shown in Figure 5.

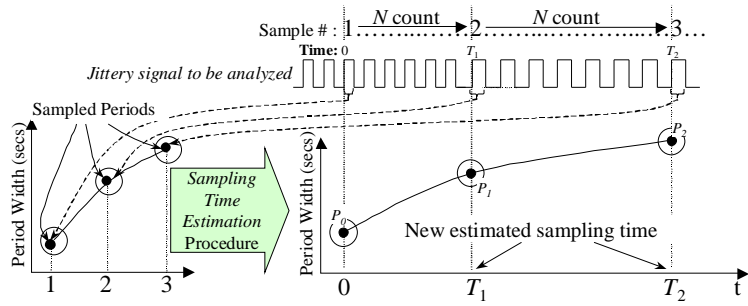

Figure 5 Estimate the sampled time of each measured Period
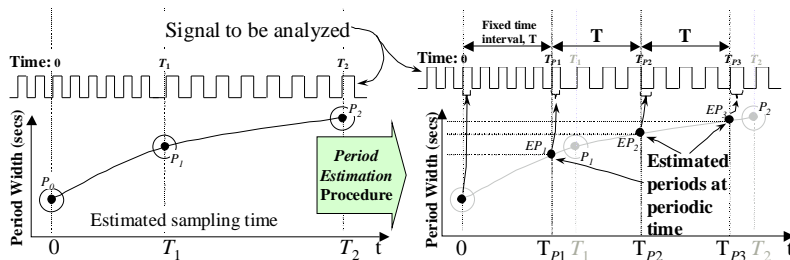

Figure 6 Estimate the signal periods at periodic time interval

The next procedure is to estimate the width of the signal period that occurs at periodic time intervals $P_{n}$ to analyze the jitter spectrum. Since we have generated a list of measured signal periods with their respective time incidences $T_{n}$, estimating a list of signal periods that occur periodically at $P_{n}$ can be easily accomplished through interpolation using the Period Estimation procedure as shown in Figure 6.

For simplicity, a random jitter component was not included in Figure 5 and Figure 6. After generating a list of estimated signal periods at periodic intervals, the technique performs Fast Fourier Transformation (FFT) on the list to extract any sinusoidal/periodic jitter that may be found in the signal. The details of each algorithm utilized in the spectral analysis technique will be presented in Section IV.

B. Implementation of Signal Period Sampling

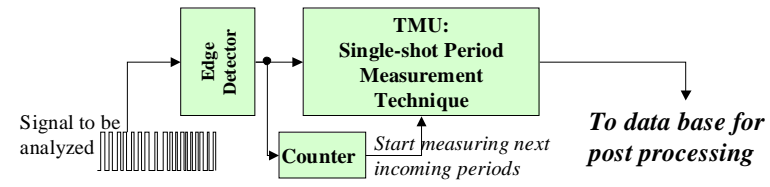

Figure 7 Block diagram for the technique implementation

In order to measure the signal period at every $N$ count, the implementation would require some kind of counter to trigger the TMU. Figure 7 shows how the sampling of signal periods may be implemented. However, implementing a counter operating at the multi-gigahertz range would be very challenging.

In order to ease the counter implementation, we suggest the count $N$ to be equal to $2^{n}$, where $n$ is any positive integer number. In this way, the counter can be implemented using $n$ clock dividers, which are much simpler and cost-effective than implementing an arbitrary counter operating at the multi-gigahertz range. With the sampling of the signal period at an interval of $N$ periods resolved, the key remaining issue for the deployment of the technique is the implementation of the TMU.

Since the TMU may require some time to determine the signal period measurement, the count value $N$ is selected so that the time taken to complete the count should be longer than the required time for the TMU to perform the measurement.

\section{The SPECTRAL ANAlysis TeChNiQUe}

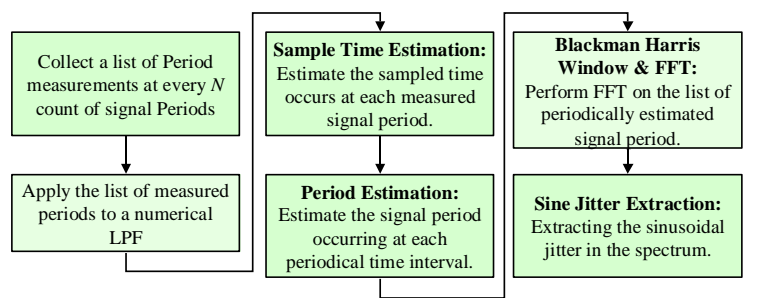

Figure 8 Detailed technique flow

This section presents the details of the spectral analysis technique. The general form of signal jitter is represented by the sum of a Gaussian random process and multiple sinusoidal processes as follows:

$$
J_{\text {sig }}=P_{\text {random }}(\sigma)+\sum_{i=1}^{n} a_{i} \sin \left(\omega_{i} t\right)
$$

Because of the high frequency signals introduced by the Gaussian random process, the Sample Time Estimation procedure becomes inaccurate. Hence, we apply the list of sampled periods to a numerical Low-Pass Filter (LPF), as part of the technique flow shown in Figure 8, to remove the high-frequency signal jitter from the list. The bandwidth of the LPF must be larger than the highest frequency of the sinusoidal jitters. This requirement is to avoid attenuation of sinusoidal jitters whose frequency is higher than the LPF bandwidth if the latter is not chosen properly. Hence, some knowledge of the deterministic jitter frequency helps improve the accuracy of the extracted jitter spectrum information.

\section{A. Sample Time Estimation Procedure}

The Sample Time Estimation procedure assumes that the lengths of the $(N-1)$ signal periods between the two sampled periods $P_{1}$ and $P_{2}$, can be estimated by linear interpolation as depicted in Figure 9. Because the sampling of signal periods is based on signal periods count $N$ rather than on absolute timing, the sampling time $T_{2}$ of the current period $P_{2}$, will be the sampling time $T_{1}$ of the previous sampled period $P_{1}$ plus the sum of the lengths of all periods that occur between $P_{1}$ and $P_{2}$ as depicted in Figure 9 . Hence, the current sampling time $T_{n}$ can be expressed in terms of the count $N$, the previous sampling time $T_{n-l}$, the current sampled period width $W_{P_{n}}$, and previous sampled period $W_{P_{n-1}}$, as follows:

$$
T_{n}=T_{n-1}+\frac{W_{P_{n-1}}(N+1)+W_{P_{n}}(N-1)}{2}
$$

This equation could estimate the sampling time of each sampled period $P_{n}$, thus allowing us to approximate the signal 
periods that occur periodically using the Period Estimation procedure.

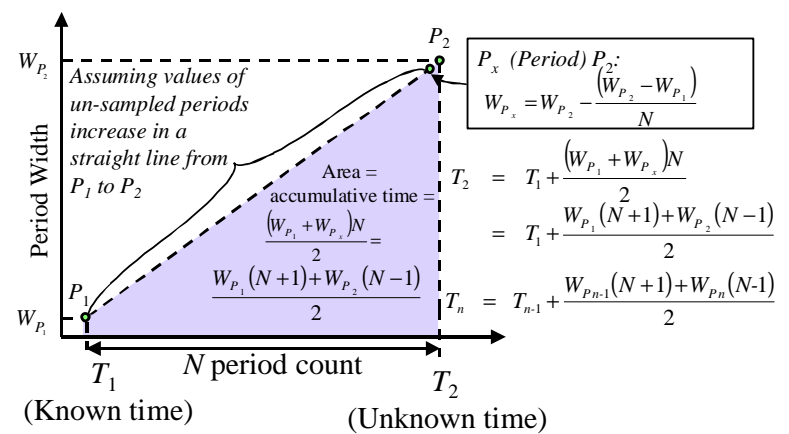

Figure 9 Sample time estimation

\section{B. Period Estimation Procedure}

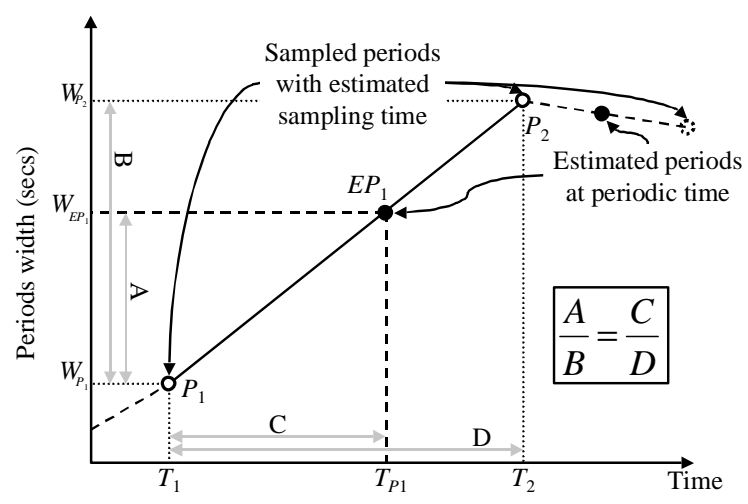

Figure 10 Estimating signal periods at periodic time

With the same assumption mentioned in the previous section, we could use linear interpolation to estimate the signal periods that occur at periodic times shown in Figure 10. The width $W_{E P_{1}}$ of the estimated signal period $E P_{1}$, can be determined using following equation:

$$
W_{E P 1}=W_{P_{1}}+\left(T_{P_{1}}-T_{1}\right) \frac{W_{P_{2}}-W_{P_{1}}}{T_{2}-T_{1}}
$$

where $T_{P 1}$ is the periodic time which $E P_{1}$ occurs, while $T_{1}$ and $T_{2}$ are the immediate-adjacent sampling times estimated in the previous Sampling Time Estimation procedure. $W_{P_{1}}$ and $W_{P_{2}}$ are the widths of the immediate-adjacent sampled periods $P_{1}$ and $P_{2}$ respectively. The output of this procedure is a list of estimated signal periods, which is then used for FFT to extract the peaks and frequencies of the sinusoidal jitters.

\section{Sinusoidal Jitter Extraction}

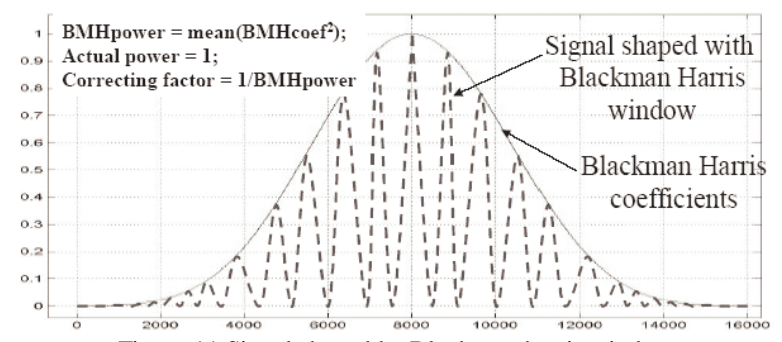

Figure 11 Signal shaped by Blackman-harris window

To reduce the frequency leakage effect when performing FFT on finite samples, some window techniques would be required [22]. We use the minimum 4-term Blackman-harris window [23] to reduce the frequency leakage. Figure 11 shows a sinusoidal signal shaped by the window's coefficients. Note that signal shaping reduces the original signal power by a factor equivalent to the mean of the coefficients' square. Hence, the spectrum power is corrected by the same factor after the FFT is performed.

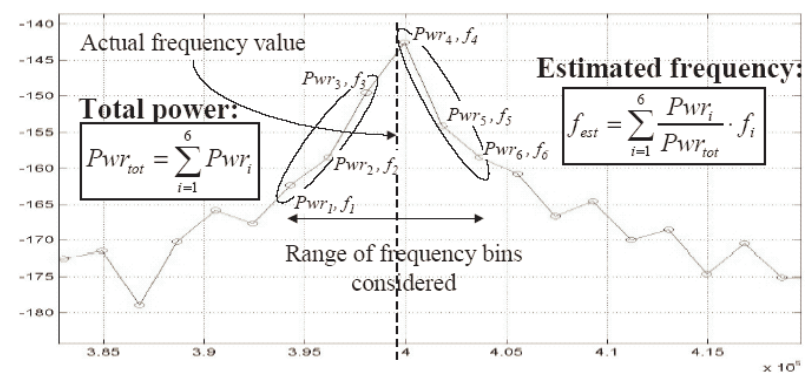

Figure 12 Estimation of the sinusoid jitter frequency

One other effect of any window technique is the spreading of signal power to its side bins. Hence, when computing the frequency and the peak of the sinusoidal jitters, a range of frequency bins adjacent to the peaks must be considered. Figure 12 shows one of the sinusoid peaks found in the spectrum. The sinusoidal jitter total power $P w r_{t o t}$ can be computed as the sum of power in the predetermined frequency range as shown in Figure 12. The sinusoid jitter frequency $f_{\text {est }}$ can be computed as the sum of weighted frequencies considered in the frequency range shown in one of the Figure 12 equations. Each frequency within the range is weighted by its associate power normalized to the previously computed total power.b

\section{Random Jitter Extraction}

The random jitter variance is equivalent to the total noise power present in the signal period spectrum. Since the signal periods were LPF'ed before the Sampling Time Estimation procedure, the high-frequency noise power is attenuated. In order to restore a flat noise spectrum, the filtered period spectrum is reshaped by the inverse transfer function of LPF. Both the filtered and the reshaped spectrums are shown in Figure 13. Because of the unstable inverse transfer function, the reshaped spectrum has very large high-frequency power. Hence, only the flat frequency range $A$ could be used to estimate the total noise power $P_{N S E_{-} t o t}$ as shown in Figure 13. $P_{N S E \_A}$ is the total noise power in the frequency range $A$, whereas $B$ is the total frequency range.

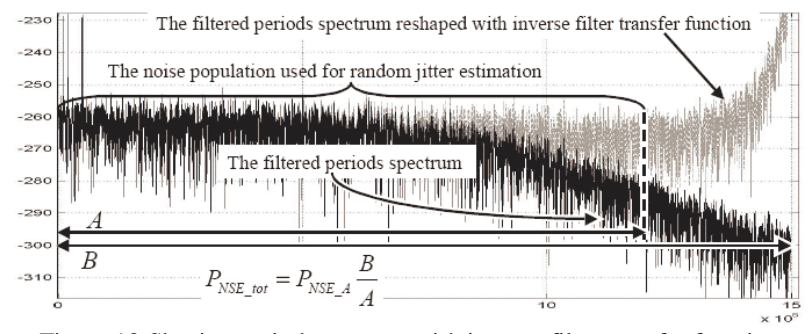

Figure 13 Shaping period spectrum with inverse filter transfer function

\section{EXPERIMENT SETUP AND RESULT}

An experiment was set up in the MATLAB simulation to validate the spectral extraction technique. As shown in Figure 14, a $3 \mathrm{G} \mathrm{Hz}$ signal is generated with a jitter characteristic whose function is the sum of three sinusoidal processes and a random Gaussian distribution process. Sum of sinusoidal processes peaks is 0.1 Unit Interval (UI - equivalent to the 
signal period). The random process has a standard deviation of UI/( $2 * 14.069)$. The sinusoidal frequencies are in the $100 \mathrm{~K}$ $\mathrm{Hz}$ frequency range, and their maximum is $500 \mathrm{~K} \mathrm{~Hz}$. The count $N$ is set to 128 making the periods' average sampling frequency equivalent to $23.4 \mathrm{M} \mathrm{Hz}(3 \mathrm{G} / 128 \mathrm{~Hz})$. This sampling frequency allows the technique to extract any sinusoidal jitter with frequency below $11.7 \mathrm{M} \mathrm{Hz}$. Figure 15 shows the transient plot of the signal periods after sampling at every 128-period count.

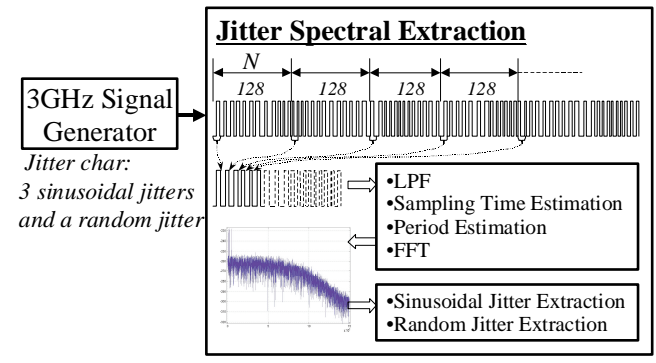

Figure 14 Experiment Setup

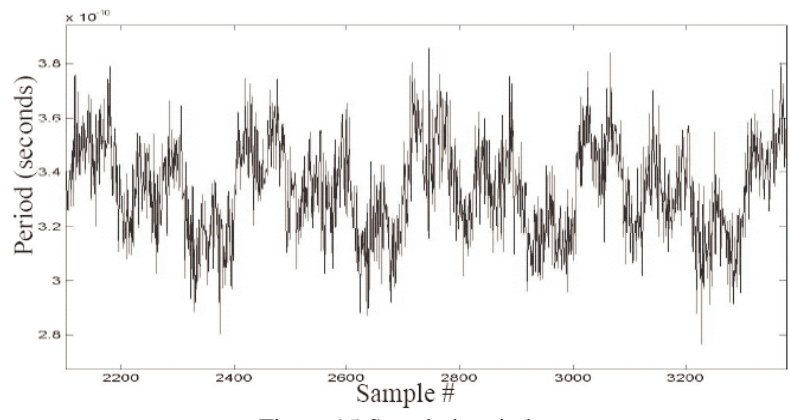

Figure 15 Sampled periods

Table 1 Experiment results

\begin{tabular}{|c|c|c|c|c|c|c|}
\hline $\begin{array}{l}\text { Sample } \\
\text { Length }\end{array}$ & $\begin{array}{l}\text { Avg. Freq } \\
\text { Err }(\%)\end{array}$ & $\begin{array}{l}3 \sigma \text { Freq } \\
\text { Err }(\%)\end{array}$ & $\begin{array}{l}\text { Avg. Peak } \\
\text { Err (\%) }\end{array}$ & $\begin{array}{l}3 \sigma \text { Peak } \\
\text { Err }(\%)\end{array}$ & $\begin{array}{l}\text { Avg. Rand Std } \\
\text { Err }(\%)\end{array}$ & $\begin{array}{c}3 \sigma \text { Rand } \\
\text { Std Err }(\%)\end{array}$ \\
\hline $2^{11}$ & 0.02 & 1.28 & 0.88 & 17.2 & -6.97 & 7.9 \\
\hline $2^{12}$ & 0.01 & 0.55 & 0.82 & 11.8 & -6.59 & 6.93 \\
\hline $2^{13}$ & -0.004 & 0.27 & 0.47 & 9.2 & -6.88 & 4.98 \\
\hline $2^{14}$ & -0.003 & 0.16 & -0.33 & 5.6 & -6.37 & 3.79 \\
\hline $2^{15}$ & -0.001 & 0.08 & -0.14 & 3.7 & -6.08 & 2.22 \\
\hline $2^{16}$ & 0.002 & 0.07 & -0.33 & 3.2 & -5.91 & 1.79 \\
\hline $2^{17}$ & 0.001 & 0.05 & -0.38 & 2.4 & -5.57 & 1.38 \\
\hline
\end{tabular}

The experiment was conducted using seven different sample lengths shown in the first column of Table 1. For each sample length, the experiment was conducted a hundred times to assess the technique's accuracy. The second column indicates the technique's average estimation error for the three sinusoidal process frequencies corresponding to the first column's sample length. The third column shows the technique's three-sigma $3 \mathrm{~s}$ estimation error for the sinusoid frequencies. Likewise, the fourth column shows the average estimation for the sinusoidal process peaks, while the fifth column shows the technique's $3 \mathrm{~s}$ estimation error for the sinusoid peaks. The sixth column shows the average error for estimating the random process standard deviation, whereas the seventh column indicates the technique's 3s estimation error for the random process standard deviation. As one might expect, the larger sample length yields smaller deviation in the technique estimation, and hence achieves higher estimation accuracy.

\section{Conclusion}

Any spectral analysis scheme requires accurate sampling and measurement mechanisms to achieve accurate results. In this paper, we propose a signal period spectral extraction technique that does not require an ideal sampling clock; thus it obviates the need for an ideal sampling mechanism. Although the technique still relies on the period measurement circuitry to measure the signal periods, any unwanted jitter introduced by that circuitry could also be extracted by the proposed technique during its calibration phase. Such unwanted jitter can then be removed from the extracted spectrum of the signal periods, thus further increase the technique's accuracy. Combining with an on-chip singleshot period measurement technique, this technique could be implemented for on-chip period/jitter spectral analysis.

\section{REFERENCE}

1 M. Lauterbach, and T. Wey, "Analyze jitter to improve high-speed design," In IEEE Spectrum, Vol. 37, pp 62 - 67, July 2000.

2 W. Dalal and D. Rosenthal, "Measuring of High Speed Data Channels Using Undersampling Techniques," In Proc. of International Test Conference, 1998, pp $819-23$.

3 M. Li and et al, "A New Method for Jitter Decomposition through its Distribution Tail Fitting," In Proc. of International Test Conference, 1999, pp $788-94$.

4 S. Jie, M. Li, and J Wilstrup, "A demonstration of deterministic jitter (DJ) deconvolution," In Proc. of Instrumentation and Measurement Technology Conference, 2002, pp 293 - 8.

5 Y. Cai, B. Laquai, and K. Luchman, "Jitter Testing for Gigabit Serial Communication Transceivers," In IEEE Design \& Test of Computers, Vol. 19, pp 66 -74, Jan. - Feb. 2002.

6 Y. Cai, et al, "Jitter Testing for Multi-Gigabit Backplane SerDes Techniques to decompose and Combine Various Types of Jitter," In Proc. of International Test Conference, 2002, pp $700-9$.

7 T. Yamaguchi, et al, "Extraction of Peak-to-Peak and RMS Sinusoidal Jitter Using an Analytic Signal Method," In Proc. of VLSI Test Symposium, 2000, pp 395 - 402.

8 T. Yamaguchi, et al, "Timing Jitter Measurement of 10 Gbps Bit Clock Signals using Frequency Division," In Proc. of VLSI Test Symposium, 2002, pp $207-212$.

9 M. Li, and J. Wilstrup, "On the Accuracy of Jitter Separation From Bit Error Rate Function,” In Proc. of International Test Conference, 2002, pp $710-6$.

10 J. Wilstrup, “A Method of Serial Data Jitter Analysis Using One-shot Time Interval Measurements," In Proc. of International Test Conference, 1998, pp $819-23$.

11 E. Räisänen-Ruotsalainen, et al, "Time interval measurements using time-to-voltage conversion with built-in dual-slope A/D conversion," In IEEE International Symposium on Circuits and Systems, Vol.5, pp 2573 - 2576, June 1991.

12 M. Hsiao, et al, "A low-cost CMOS time interval measurement core," In IEEE International Symposium on Circuits and Systems, Vol. 4, pp 190 - 193, May 2001.

13 E Räisänen-Ruotsalainen, “A low-power CMOS time-to-digital converter," In IEEE Journal of Solid-State Circuits, Vol. 30, pp 984 90, Sept. 1995.

14 P. Chen, and S.-I Liu, "A cyclic CMOS time-to-digital converter with deep sub-nanosecond resolution," In Proc. of IEEE Custom Integrated Circuits, May 1999, pp 605 - 8.

15 A. Chan, and G. Roberts, "A synthesizable, fast and high-resolution timing measurement device using a component-invariant vernier delay line," In Proc. of International Test Conference, Oct 2001 pp 858 - 67.

16 N. Abaskharoun, G. Roberts, "Circuits for on-chip sub-nanosecond signal capture and characterization," In Proc. of IEEE Custom Integrated Circuits, May 2001, pp $251-4$.

17 D. Chu, "Double vernier time interval measurement using triggered phase-locked oscillators," US Patent \#4164648 assigned to HewlettPackard Company, Aug 141979. 
18 A. Frisch and T. Rinderknecht, "Jitter measurement system and method," US Patent \#6,295,315 assigned to Fluence Technologies, Sep 252001.

19 S. Tabatabaei, and A. Ivanov, "An embedded core for sub-picosecond timing measurements," In Proc. of International Test Conference, Oct. 2002, pp 129 - 137.

20 A. Chan, G. Roberts, "A deep sub-micron timing measurement circuit using a single-stage Vernier delay line," In Proc. of IEEE Custom Integrated Circuits Conference, May 2002, pp 77 - 80.

21 M. Gorbics, et al, "A high resolution multihit time to digital converter integrated circuit," In IEEE Transactions on Nuclear Science, Vol. 44, pp 379 -384, June 1997.

22 S. Orfanidis, Introduction To Signal Processing. Prentice Hall International Inc., 1996.

23 F. Harris, "On the Use of Windows for Harmonic Analysis with the Discrete Fourier Transform." In Proc. of the IEEE, Vol. 66, pp. 51-84, Jan. 1978. 